\title{
Soil Fertility and Yield of Rice During Two Season on Organic and Inorganic Fertilized
}

\author{
Sugiyanta ${ }^{1}$, Isna Tustiyani ${ }^{2}$, Maya Melati ${ }^{3}$, Agus Rachman Nurrizki ${ }^{4}$ \\ \{mr_sugiyanta@yahoo.co.id ${ }^{1}$, isnatustiyani@gmail.com² ${ }^{2}$ maya_melati05@yahoo.com ${ }^{3}$, \\ nurrizki@yahoo.com $\left.{ }^{4}\right\}$ \\ Department of Agronomy and Horticulture, IPB University, Indonesia ${ }^{1}$ \\ Agrotechnology Study Program, Faculty of Agriculture, Universitas Garut, Indonesia ${ }^{2}$ \\ Alumni of Department of Agronomy and Horticulture, IPB University, Indonesia ${ }^{3}$
}

\begin{abstract}
The objective of the research was to investigate the soil fertility and yield of rice during two season on organic and inorganic fertilized. The experiment was conducted at rice field in Karawang and Bogor, West Java. The experiment used one factor in randomized block design consisted of three replications with 13 treatments. The first 6 treatments were $0,2,4,6,8,10$ ton organic fertilizer ha ${ }^{-1}$, and the other 6 treatments were $0,2,4,6,8,10$ ton organic fertilizer ha $\mathrm{a}^{-1}$ combined with $2 \mathrm{~L}$ biological fertilizer ha $\mathrm{h}^{-1}$ and as control treatment was application of anorganic fertilizer with the rate of $400 \mathrm{~kg}$ NPK (30-6-8) ha1. Plot size was $15 \mathrm{~m}$ x $10 \mathrm{~m}$, with a double row spacing. The results showed that the variables on first season such as number of tillers and leaf color in inorganic fertilizer treatment was higher when compared with the organic fertilizers. Soil fertility on organic fertilizer treatment increased in the second season and yield decreased in the second season.
\end{abstract}

Keywords: inorganic, organic, soil fertility, two season, yield.

\section{Introduction}

The use of inorganic fertilizers continuously resulting in negative effects on the soil and the environment [1] as resulting in paddy soil becomes solid, decreasing porosity, soil density (bulk density) increases, and declining soil fertility [2] and increased pest and disease [3]. Soils condition and its health status was vital part for the agricultural production [4]. The decline in soil fertility can be seen from declining of soil organic matter and $\mathrm{pH}$ [5]. Public awareness of the negative impacts of excessive inorganic fertilizer, the society is slowly making the transition to organic farming.

One of the components of organic farming is organic and biological fertilizers. Organic fertilizers play a role in improving and enhancing soil fertility [6], good chemical, physical, and biological of soil [7] and it can give macronutrients and micronutrients [8]. Biological fertilizers 
play a role in influencing the availability of macro and micro nutrients, nutrient uptake, enzyme system performance, increase metabolism, growth and yield [9]. One of the advantages of biological fertilizer that can improve nutrient uptake, if fertilizer is combined with organic fertilizers is expected to reduce the dose of organic fertilizer in the next planting season. The goal of this experiment was to study the soil fertility and yield of rice for two seasons on organic and inorganic fertilized.

\section{Materials and methods}

Field trials conducted in January-September 2012 at Karawang Wetan, Karawang Wetan District of Karawang Timur, West Java, Indonesia at an altitude of 15 meters above sea level during the two growing seasons. Soil analysis carried out in the Laboratory of Chemistry and Soil Fertility Department of Soil Science and Land Resources IPB. This experiment uses a single factor design with 13 treatments were arranged in a randomized block design with three replications. Treatment consisted of 13 treatments were $0,2,4,6,8,10$ tons of organic fertilizer ha-1, and $0,2,4,6,8,10$ tons of organic fertilizer ha-1 each plus $2 \mathrm{~L}$ fertilizer biological treatment ha-1 and $400 \mathrm{~kg}$ NPK inorganic fertilizer (30-6-8) ha-1.

Experimental plot size was $15 \mathrm{~m} \times 10 \mathrm{~m}$. The Menthik Wangi seeds sowing in seedbed, after 10 days transferred to the experimental plots with 2 plant per hole. The spacing used a double row space (legowo 2:1) $(25 \mathrm{~cm} \times 15 \mathrm{~cm} \times 50 \mathrm{~cm})$. Organic fertilizer given before planting and biological fertilizers sprayed through the leaves at the age of 4 and 6 week after planting (WAP) respectively at 50\% dose, and the NPK fertilizer given at planting time.

The parameters observe were soil analysis $(\mathrm{pH}$, organic $\mathrm{C}, \mathrm{N}, \mathrm{P}$, and $\mathrm{K})$, number of tillers, leaf color, number of productive tillers, 1000 grain weight, the panicle length, number of grains per panicle, percent empty grains, and yield. The statistical data analysis using analysis of variance, if the level of $\alpha=0.05$ level indicates a real effect, then continued by DMRT. Data calculations were performed using SAS (Statistical Analysis System).

\section{Result}

The results of the soil analysis showed that the soil before the experiment has a neutral $\mathrm{pH}$, low levels of organic C, N, K level, and very high levels of P (Table 1). Low organic C content was in line with reportson organic treatment [10]. Table 1 showed that during the first and second growing season decline in soil $\mathrm{pH}$ values from neutral at the beginning of the experiment becomes slightly acid at the first planting season and acid in the second growing season. Organic $\mathrm{C}$ levels increased during the initial trial experiments, low organic $\mathrm{C}$ at first season and moderate organic $\mathrm{C}$ the second season. The organic fertilizer will increase the organic C content [12]. Soil $\mathrm{N}$ levels also increased during the two growing seasons. Soil $\mathrm{P}$ levels decreased in the first season to the second season. 
Table 1. Analysis of the soil at different fertilizer treatments

\begin{tabular}{|c|c|c|c|c|c|}
\hline Treatment & pH & C-org(\%) & N-total(\%) & $\begin{array}{c}\text { P Olsen } \\
\text { (ppm) }\end{array}$ & $\mathbf{K}(\mathbf{p p m})$ \\
\hline \multirow[t]{2}{*}{ Before treatment } & $7.4(\mathrm{~N})$ & $1.84(\mathrm{~L})$ & $0.17(\mathrm{~L})$ & $61.5(\mathrm{VH})$ & $151(\mathrm{VL})$ \\
\hline & \multicolumn{5}{|c|}{ The end of the first planting season } \\
\hline 0 ton ha- ${ }^{1} \mathrm{OF}$ & $5.7(\mathrm{SA})$ & $1.39(\mathrm{~L})$ & $0.14(\mathrm{~L})$ & $78(\mathrm{VH})$ & $78(\mathrm{VL})$ \\
\hline 2 ton ha- 1 OF & 5.7 (SA) & $1.34(\mathrm{~L})$ & $0.15(\mathrm{~L})$ & $40(\mathrm{VH})$ & $76(\mathrm{VL})$ \\
\hline 4 ton ha- 1 OF & $5.4(\mathrm{~A})$ & $1.39(\mathrm{~L})$ & $0.16(\mathrm{~L})$ & $0(\mathrm{VL})$ & $78(\mathrm{VL})$ \\
\hline 6 ton ha- 1 OF & $5.2(\mathrm{~A})$ & $1.41(\mathrm{~L})$ & $0.15(\mathrm{~L})$ & $0(\mathrm{VL})$ & $76(\mathrm{VL})$ \\
\hline 8 ton ha- 1 OF & $5.6(\mathrm{SA})$ & $1.31(\mathrm{~L})$ & $0.13(\mathrm{~L})$ & $42(\mathrm{VH})$ & $103(\mathrm{VL})$ \\
\hline 10 ton ha- $1 \mathrm{OF}$ & $6.3(\mathrm{SA})$ & $1.13(\mathrm{~L})$ & $0.13(\mathrm{~L})$ & $34(\mathrm{VH})$ & 47 (VL) \\
\hline 0 ton ha- $1 \mathrm{OF}+\mathrm{BF}$ & $5.8(\mathrm{SA})$ & $1.28(\mathrm{~L})$ & $0.14(\mathrm{~L})$ & $55(\mathrm{VH})$ & $78(\mathrm{Vl})$ \\
\hline 2 ton ha- $1 \mathrm{OF}+\mathrm{BF}$ & 5.7 (SA) & $1.25(\mathrm{~L})$ & $0.13(\mathrm{~L})$ & $52(\mathrm{VH})$ & $63(\mathrm{VL})$ \\
\hline 4 ton ha- $1 \mathrm{OF}+\mathrm{BF}$ & $5.6(\mathrm{SA})$ & $1.36(\mathrm{~L})$ & $0.16(\mathrm{~L})$ & $49(\mathrm{VH})$ & $76(\mathrm{Vl})$ \\
\hline 6 ton ha- $1 \mathrm{OF}+\mathrm{BF}$ & $5.5(\mathrm{~A})$ & $1.33(\mathrm{~L})$ & $0.16(\mathrm{~L})$ & 49 (VH) & $92(\mathrm{Vl})$ \\
\hline 8 ton ha- $1 \mathrm{OF}+\mathrm{BF}$ & 5.7 (SA) & $1.64(\mathrm{~L})$ & $0.18(\mathrm{~L})$ & $73(\mathrm{VH})$ & $151(\mathrm{VL})$ \\
\hline 10 ton ha- $1 \mathrm{OF}+\mathrm{BF}$ & $5.3(\mathrm{~A})$ & $1.32(\mathrm{~L})$ & $0.14(\mathrm{~L})$ & $0(\mathrm{VL})$ & 89 (VL) \\
\hline \multirow[t]{2}{*}{ Inorganic } & $5.6(\mathrm{SA})$ & $1.28(\mathrm{~L})$ & $0.13(\mathrm{~L})$ & $43(\mathrm{VH})$ & $63(\mathrm{VL})$ \\
\hline & \multicolumn{4}{|c|}{ The end of the second planting season } & \\
\hline 0 ton ha- $1 \mathrm{OF}$ & $5.17(\mathrm{~A})$ & $2.26(\mathrm{M})$ & $0.21 \mathrm{ab}(\mathrm{M})$ & $11.17 \mathrm{abc}(\mathrm{L})$ & $97.50 \mathrm{bc}(\mathrm{VL})$ \\
\hline 2 ton ha- $1 \mathrm{OF}$ & $5.20(\mathrm{~A})$ & $1.72(\mathrm{~L})$ & $0.17 \mathrm{ab}(\mathrm{L})$ & $9.03 \mathrm{bcd}(\mathrm{VL})$ & $\begin{array}{l}102.09 \mathrm{abc} \\
(\mathrm{VL})\end{array}$ \\
\hline 4 ton ha- 1 OF & $5.0(\mathrm{~A})$ & $1.83(\mathrm{~L})$ & $0.19 \mathrm{ab}(\mathrm{L})$ & 7.90cd (VL) & $\begin{array}{l}120.50 \mathrm{abc} \\
(\mathrm{VL})\end{array}$ \\
\hline 6 ton ha- 1 OF & $5.00(\mathrm{~A})$ & $2.76(\mathrm{M})$ & $0.25 \mathrm{a}(\mathrm{M})$ & $7.40 \mathrm{~d}(\mathrm{VL})$ & $\begin{array}{l}135.88 \mathrm{abc} \\
(\mathrm{VL})\end{array}$ \\
\hline 8 ton ha- 1 OF & $5.07(\mathrm{~A})$ & $2.10(\mathrm{M})$ & $0.20 \mathrm{ab}(\mathrm{L})$ & $13.30 \mathrm{a}(\mathrm{L})$ & $\begin{array}{c}113.83 \mathrm{abc} \\
(\mathrm{VL})\end{array}$ \\
\hline 10 ton ha- $1 \mathrm{OF}$ & $5.20(\mathrm{~A})$ & $1.99(\mathrm{~L})$ & $0.19 \mathrm{ab}(\mathrm{L})$ & 12.17ab (L) & $88.42 \mathrm{c}(\mathrm{VL})$ \\
\hline 0 ton ha- $1 \mathrm{OF}+\mathrm{BF}$ & $5.30(\mathrm{~A})$ & $1.68(\mathrm{~L})$ & $0.16 \mathrm{ab}(\mathrm{L})$ & 12.37ab (L) & $82.18 \mathrm{c}(\mathrm{VL})$ \\
\hline 2 ton ha- $1 \mathrm{OF}+\mathrm{BF}$ & $5.37(\mathrm{~A})$ & $1.93(\mathrm{~L})$ & $0.19 \mathrm{ab}(\mathrm{L})$ & 11.90ab (L) & $90.25 \mathrm{c}(\mathrm{VL})$ \\
\hline 4 ton ha- $1 \mathrm{OF}+\mathrm{BF}$ & $5.13(\mathrm{~A})$ & $1.96(\mathrm{~L})$ & $0.22 \mathrm{ab}(\mathrm{M})$ & $13.03 \mathrm{a}(\mathrm{L})$ & $\begin{array}{c}145.53 \mathrm{ab} \\
(\mathrm{VL})\end{array}$ \\
\hline 6 ton ha- $1 \mathrm{OF}+\mathrm{BF}$ & $5.03(\mathrm{~A})$ & $1.61(\mathrm{~L})$ & $0.16 b(\mathrm{~L})$ & 11.43ab (L) & $\begin{array}{c}124.03 \mathrm{abc} \\
(\mathrm{VL})\end{array}$ \\
\hline 8 ton ha- $1 \mathrm{OF}+\mathrm{BF}$ & $5.20(\mathrm{~A})$ & $1.83(\mathrm{~L})$ & $0.17 \mathrm{ab}(\mathrm{L})$ & 12.13ab (L) & $\begin{array}{l}101.34 \mathrm{abc} \\
\quad(\mathrm{VL})\end{array}$ \\
\hline 10 ton ha- $1 \mathrm{OF}+\mathrm{BF}$ & $5.43(\mathrm{~A})$ & 1.74(L) & $0.17 \mathrm{ab}(\mathrm{L})$ & $6.33 \mathrm{~d}(\mathrm{VL})$ & $151.75 \mathrm{a}(\mathrm{VL})$ \\
\hline Inorganic & $5.20(\mathrm{SA})$ & $2.20(\mathrm{M})$ & $0.22 \mathrm{ab}(\mathrm{M})$ & $12.633 \mathrm{ab}(\mathrm{L})$ & $70.08 \mathrm{c}(\mathrm{VL})$ \\
\hline
\end{tabular}

- $\quad$ OF: organic fertilizer, BF: 2 L ha-1 biological fertilizer

- $\quad$ (SA): slightly acid, (A): Acid, (L); low, (VL): very low, (M): Medium, (VH): very high, based on the criteria of soil analysis Soil Research Center (2008).

- Values followed by different letters within a column are significantly different at 95\% DMRT. 
The number of tillers in the first season on the treatment of inorganic fertilizers is highest one, but in the second season, the number of tillers of organic fertilized equivalent to inorganic fertilizer treatment (Table 2). This is presumably due to the role of organic fertilizers on the plant will begin to appear on the next season effect [7].

Table 2. Number of tiller at different fertilizer treatments

\begin{tabular}{|c|c|c|c|c|c|c|}
\hline \multirow{3}{*}{ Treatment } & \multicolumn{6}{|c|}{ Number of tillers (week after planting) } \\
\hline & 3 & 4 & 5 & 6 & 7 & 8 \\
\hline & \multicolumn{6}{|c|}{ First season } \\
\hline 0 ton ha- ${ }^{1} \mathrm{OF}$ & $20.4 \mathrm{ab}$ & $30.1 \mathrm{ab}$ & $29.7 \mathrm{ab}$ & $29.1 \mathrm{~b}$ & $27.5 \mathrm{ab}$ & $19.3 \mathrm{a}$ \\
\hline 2 ton ha- ${ }^{1} \mathrm{OF}$ & $20.9 \mathrm{ab}$ & $28.2 \mathrm{ab}$ & $27.5 b$ & $27.1 \mathrm{~b}$ & $25.9 \mathrm{ab}$ & $18.7 \mathrm{a}$ \\
\hline 4 ton ha- ${ }^{1} \mathrm{OF}$ & $17.4 \mathrm{~b}$ & $28.7 \mathrm{ab}$ & $27.5 b$ & $25.4 \mathrm{~b}$ & $25.2 \mathrm{ab}$ & $18.4 \mathrm{a}$ \\
\hline 6 ton ha- ${ }^{1} \mathrm{OF}$ & $16.9 \mathrm{~b}$ & $27.5 b$ & $24.9 b$ & $28.6 b$ & $27.2 \mathrm{ab}$ & $18.1 \mathrm{a}$ \\
\hline 8 ton ha- ${ }^{1} \mathrm{OF}$ & $18.1 \mathrm{~b}$ & $26.3 b$ & $25.8 \mathrm{~b}$ & $25.2 \mathrm{~b}$ & $25.9 \mathrm{ab}$ & $18.1 \mathrm{a}$ \\
\hline 10 ton ha- $^{-1}$ OF & $17.6 \mathrm{~b}$ & $29.7 \mathrm{ab}$ & $26.5 b$ & $27.0 \mathrm{~b}$ & $25.3 \mathrm{ab}$ & $19.1 \mathrm{a}$ \\
\hline 0 ton ha- ${ }^{1} \mathrm{OF}+\mathrm{BF}$ & $18.2 b$ & $29.4 \mathrm{ab}$ & $26.8 b$ & $27.1 \mathrm{~b}$ & $29.2 \mathrm{a}$ & $20.0 \mathrm{a}$ \\
\hline 2 ton ha- ${ }^{1} \mathrm{OF}+\mathrm{BF}$ & $19.6 \mathrm{ab}$ & $26.4 b$ & $25.5 b$ & $25.5 b$ & $27.9 \mathrm{ab}$ & $18.1 \mathrm{a}$ \\
\hline 4 ton ha- ${ }^{1} \mathrm{OF}+\mathrm{BF}$ & $17.4 \mathrm{~b}$ & $27.0 \mathrm{~b}$ & $24.1 \mathrm{~b}$ & $24.9 \mathrm{~b}$ & $23.6 b$ & $17.7 \mathrm{a}$ \\
\hline 6 ton ha- ${ }^{1} \mathrm{OF}+\mathrm{BF}$ & $19.1 \mathrm{~b}$ & $29.3 \mathrm{ab}$ & $28.8 \mathrm{ab}$ & $29.1 b$ & $26.4 \mathrm{ab}$ & $18.6 \mathrm{a}$ \\
\hline 8 ton ha- ${ }^{1} \mathrm{OF}+\mathrm{BF}$ & $16.8 b$ & $27.5 b$ & $24.6 \mathrm{~b}$ & $25.7 \mathrm{~b}$ & $24.3 \mathrm{ab}$ & $17.7 \mathrm{a}$ \\
\hline 10 ton ha- ${ }^{1} \mathrm{OF}+\mathrm{BF}$ & $19.1 \mathrm{~b}$ & $27.1 \mathrm{~b}$ & $26.1 \mathrm{~b}$ & $27.3 b$ & $25.5 \mathrm{ab}$ & $18.5 \mathrm{a}$ \\
\hline \multirow[t]{2}{*}{ Inorganic } & $25.3 \mathrm{a}$ & $35.6 \mathrm{a}$ & $33.8 \mathrm{a}$ & $34.4 \mathrm{a}$ & $29.3 \mathrm{a}$ & $20.8 \mathrm{a}$ \\
\hline & \multicolumn{6}{|c|}{ Second season } \\
\hline 0 ton ha- ${ }^{1} \mathrm{OF}$ & $13.7 \mathrm{a}$ & $22.1 \mathrm{a}$ & $27.2 \mathrm{a}$ & $24.8 \mathrm{a}$ & $21.9 \mathrm{a}$ & $19.1 \mathrm{a}$ \\
\hline 2 ton ha- ${ }^{1} \mathrm{OF}$ & $8.9 \mathrm{a}$ & $18.7 \mathrm{a}$ & $19.9 \mathrm{a}$ & $21.6 \mathrm{a}$ & $19.4 \mathrm{a}$ & $16.5 \mathrm{a}$ \\
\hline 4 ton ha- ${ }^{1} \mathrm{OF}$ & $10.5 \mathrm{a}$ & $19.1 \mathrm{a}$ & $23.1 \mathrm{a}$ & $25.9 \mathrm{a}$ & $23.3 \mathrm{a}$ & $19.4 \mathrm{a}$ \\
\hline 6 ton ha- ${ }^{1} \mathrm{OF}$ & $8.7 \mathrm{a}$ & $21.6 \mathrm{a}$ & $23.4 \mathrm{a}$ & $28.1 \mathrm{a}$ & $24.5 \mathrm{a}$ & $20.8 \mathrm{a}$ \\
\hline 8 ton ha- ${ }^{1} \mathrm{OF}$ & $10.4 \mathrm{a}$ & $19.1 \mathrm{a}$ & $23.1 \mathrm{a}$ & $25.4 \mathrm{a}$ & $22.8 \mathrm{a}$ & $18.9 \mathrm{a}$ \\
\hline 10 ton ha- $^{1}$ OF & $10.0 \mathrm{a}$ & $22.0 \mathrm{a}$ & $22.9 a$ & $26.1 \mathrm{a}$ & $23.1 \mathrm{a}$ & $18.4 \mathrm{a}$ \\
\hline 0 ton ha- ${ }^{1} \mathrm{OF}+\mathrm{BF}$ & $12.1 \mathrm{a}$ & $21.1 \mathrm{a}$ & $22.5 \mathrm{a}$ & $24.0 \mathrm{a}$ & $21.1 \mathrm{a}$ & $18.1 \mathrm{a}$ \\
\hline 2 ton ha- ${ }^{1} \mathrm{OF}+\mathrm{BF}$ & $13.3 \mathrm{a}$ & $21.4 \mathrm{a}$ & $22.5 \mathrm{a}$ & $24.7 \mathrm{a}$ & $23.4 \mathrm{a}$ & $19.4 \mathrm{a}$ \\
\hline 4 ton ha- ${ }^{1} \mathrm{OF}+\mathrm{BF}$ & $12.2 \mathrm{a}$ & $23.0 \mathrm{a}$ & $25.5 \mathrm{a}$ & $27.8 \mathrm{a}$ & $22.7 \mathrm{a}$ & $26.7 \mathrm{a}$ \\
\hline 6 ton ha- ${ }^{1} \mathrm{OF}+\mathrm{BF}$ & $10.5 \mathrm{a}$ & $20.2 \mathrm{a}$ & $33.5 \mathrm{a}$ & $26.4 \mathrm{a}$ & $22.0 \mathrm{a}$ & $17.3 \mathrm{a}$ \\
\hline 8 ton ha- ${ }^{1} \mathrm{OF}+\mathrm{BF}$ & $8.1 \mathrm{a}$ & $18.9 \mathrm{a}$ & $19.9 \mathrm{a}$ & $22.5 \mathrm{a}$ & $20.9 \mathrm{a}$ & $19.7 \mathrm{a}$ \\
\hline 10 ton ha- ${ }^{1} \mathrm{OF}+\mathrm{BF}$ & $12.8 \mathrm{a}$ & $21.9 \mathrm{a}$ & $22.2 \mathrm{a}$ & $25.2 \mathrm{a}$ & $22.5 \mathrm{a}$ & $19.5 \mathrm{a}$ \\
\hline Inorganic & $10.7 \mathrm{a}$ & $23.0 \mathrm{a}$ & $26.3 \mathrm{a}$ & $26.6 \mathrm{a}$ & $31.0 \mathrm{a}$ & $20.0 \mathrm{a}$ \\
\hline
\end{tabular}

Note:

- OF: organic fertilizer, BF: 2 L ha-1 biological fertilizer

- Values followed by different letters within a column are significantly different at 95\% DMRT.

In contrast to the plant height and number of tillers, leaf color variables on the first season and second season continued to show that the treatment of inorganic fertilizers have more color dark green leaves when compared with organic fertilizer treatment (Table 3). Leaf color chart 
shows the level of adequacy of nutrient nitrogen. Nitrogen is needed for growth and yield [7]. Organic and biological fertilizer suspected could not provide enough nitrogen for the plants due to organic fertilizer slow provides nutrients [12], while inorganic fertilizers was having a a high nutrient content and quickly available to plants.

Table 3. Leaf color at different fertilizer treatments

\begin{tabular}{|c|c|c|c|c|c|c|}
\hline \multirow{3}{*}{ Treatment } & \multicolumn{6}{|c|}{ Leaf color (week after planting) } \\
\hline & 3 & 4 & 5 & 6 & 7 & 8 \\
\hline & \multicolumn{6}{|c|}{ First season } \\
\hline 0 ton ha- ${ }^{1} \mathrm{OF}$ & $2.48 \mathrm{~b}$ & $2.12 \mathrm{e}$ & $2.33 b$ & $2.37 \mathrm{c}$ & $2.50 \mathrm{bc}$ & $2.57 b$ \\
\hline 2 ton ha- ${ }^{1} \mathrm{OF}$ & $2.62 b$ & $2.38 \mathrm{abcde}$ & $2.50 \mathrm{~b}$ & $2.38 \mathrm{bc}$ & $2.43 \mathrm{c}$ & $2.58 \mathrm{ab}$ \\
\hline 4 ton ha- ${ }^{1} \mathrm{OF}$ & $2.62 b$ & $2.45 \mathrm{abc}$ & $2.50 \mathrm{~b}$ & $2.50 \mathrm{~b}$ & $2.52 b$ & $2.55 b$ \\
\hline 6 ton ha- ${ }^{-1} \mathrm{OF}$ & $2.55 b$ & $2.52 \mathrm{ab}$ & $2.50 \mathrm{~b}$ & $2.42 \mathrm{bc}$ & $2.45 \mathrm{bc}$ & $2.55 b$ \\
\hline 8 ton ha- $^{-1} \mathrm{OF}$ & $2.62 b$ & $2.13 \mathrm{de}$ & $2.50 \mathrm{~b}$ & $2.37 \mathrm{c}$ & $2.50 \mathrm{bc}$ & $2.62 \mathrm{ab}$ \\
\hline 10 ton ha- ${ }^{1}$ OF & $2.57 \mathrm{~b}$ & $2.35 \mathrm{bcde}$ & $2.50 \mathrm{~b}$ & $2.47 \mathrm{bc}$ & $2.45 \mathrm{bc}$ & $2.52 b$ \\
\hline 0 ton ha- ${ }^{1} \mathrm{OF}+\mathrm{BF}$ & $2.57 \mathrm{~b}$ & $2.18 \mathrm{cde}$ & $2.50 \mathrm{~b}$ & $2.47 b c$ & $2.47 \mathrm{bc}$ & $2.62 \mathrm{ab}$ \\
\hline 2 ton ha- ${ }^{1} \mathrm{OF}+\mathrm{BF}$ & $2.57 \mathrm{~b}$ & $2.40 \mathrm{abcd}$ & $2.50 \mathrm{~b}$ & $2.38 b c$ & $2.52 \mathrm{~b}$ & $2.60 \mathrm{ab}$ \\
\hline 4 ton ha- ${ }^{1} \mathrm{OF}+\mathrm{BF}$ & $2.43 b$ & $2.52 \mathrm{ab}$ & $2.50 \mathrm{~b}$ & $2.47 b c$ & $2.50 \mathrm{bc}$ & $2.53 b$ \\
\hline 6 ton ha- ${ }^{1} \mathrm{OF}+\mathrm{BF}$ & $2.43 \mathrm{~b}$ & $2.45 \mathrm{abc}$ & $2.50 \mathrm{~b}$ & $2.50 \mathrm{~b}$ & $2.48 \mathrm{bc}$ & $2.62 \mathrm{ab}$ \\
\hline 8 ton ha- ${ }^{1} \mathrm{OF}+\mathrm{BF}$ & $2.55 \mathrm{~b}$ & $2.15 \mathrm{de}$ & $2.50 \mathrm{~b}$ & $2.50 \mathrm{~b}$ & $2.50 \mathrm{bc}$ & $2.52 b$ \\
\hline 10 ton ha- ${ }^{1} \mathrm{OF}+\mathrm{BF}$ & $2.53 b$ & $2.47 \mathrm{ab}$ & $2.50 \mathrm{~b}$ & $2.50 \mathrm{~b}$ & $2.50 \mathrm{bc}$ & $2.52 b$ \\
\hline \multirow[t]{2}{*}{ Inorganic } & $2.97 \mathrm{a}$ & $2.63 \mathrm{a}$ & $2.83 a$ & $2.87 \mathrm{a}$ & $2.70 \mathrm{a}$ & $2.67 \mathrm{a}$ \\
\hline & \multicolumn{6}{|c|}{ Second season } \\
\hline 0 ton ha- ${ }^{1} \mathrm{OF}$ & $2.0 \mathrm{~d}$ & $2.1 \mathrm{e}$ & $2.2 \mathrm{c}$ & $2.2 \mathrm{f}$ & 2.3 & $2.0 \mathrm{~b}$ \\
\hline 2 ton ha- ${ }^{1} \mathrm{OF}$ & $2.2 \mathrm{c}$ & $2.3 \mathrm{~d}$ & $2.4 \mathrm{bc}$ & $2.2 \mathrm{f}$ & 2.4 & $2.0 \mathrm{~b}$ \\
\hline 4 ton ha- $^{-1} \mathrm{OF}$ & $2.3 \mathrm{bc}$ & $2.3 \mathrm{~d}$ & $2.5 b$ & $2.5 \mathrm{bcd}$ & 2.4 & $2.0 \mathrm{~b}$ \\
\hline 6 ton ha- ${ }^{1} \mathrm{OF}$ & $2.2 \mathrm{bc}$ & $2.3 \mathrm{~d}$ & $2.5 b$ & $2.2 \mathrm{f}$ & 2.6 & $2.0 \mathrm{~b}$ \\
\hline 8 ton ha- ${ }^{1} \mathrm{OF}$ & $2.4 \mathrm{bc}$ & $2.3 \mathrm{~d}$ & $2.4 b c$ & $2.3 \mathrm{def}$ & 2.5 & $2.0 \mathrm{~b}$ \\
\hline 10 ton ha- ${ }^{1} \mathrm{OF}$ & $2.2 \mathrm{c}$ & $2.3 \mathrm{~d}$ & $2.4 \mathrm{bc}$ & $2.4 \mathrm{bcdef}$ & 2.3 & $2.0 \mathrm{~b}$ \\
\hline 0 ton ha- ${ }^{1} \mathrm{OF}+\mathrm{BF}$ & $2.4 \mathrm{bc}$ & $2.4 \mathrm{~cd}$ & $2.3 \mathrm{bc}$ & $2.4 \mathrm{bcdef}$ & 2.2 & $2.1 \mathrm{~b}$ \\
\hline 2 ton ha- ${ }^{1} \mathrm{OF}+\mathrm{BF}$ & $2.4 \mathrm{bc}$ & $2.4 \mathrm{~cd}$ & $2.4 \mathrm{bc}$ & $2.3 \mathrm{def}$ & 2.6 & $2.1 \mathrm{~b}$ \\
\hline 4 ton ha- ${ }^{1} \mathrm{OF}+\mathrm{BF}$ & $2.4 \mathrm{bc}$ & $2.6 \mathrm{~b}$ & $2.4 \mathrm{bc}$ & $2.5 \mathrm{bcd}$ & 2.6 & $2.2 b$ \\
\hline 6 ton ha- ${ }^{1} \mathrm{OF}+\mathrm{BF}$ & $2.5 \mathrm{~b}$ & $2.5 \mathrm{bc}$ & $2.4 \mathrm{bc}$ & $2.5 \mathrm{bcd}$ & 2.6 & $2.0 \mathrm{~b}$ \\
\hline 8 ton ha- ${ }^{1} \mathrm{OF}+\mathrm{BF}$ & $2.5 b$ & $2.5 \mathrm{bc}$ & $2.4 \mathrm{bc}$ & $2.6 \mathrm{~b}$ & 2.6 & $2.0 \mathrm{~b}$ \\
\hline 10 ton ha- ${ }^{1} \mathrm{OF}+\mathrm{BF}$ & $2.5 b$ & $2.5 \mathrm{bc}$ & $2.5 \mathrm{~b}$ & $2.5 \mathrm{bcd}$ & 2.7 & $2.0 \mathrm{~b}$ \\
\hline Inorganic & $2.93 \mathrm{a}$ & $2.8 \mathrm{a}$ & $3.0 \mathrm{a}$ & $2.8 \mathrm{a}$ & 2.8 & $2.8 \mathrm{a}$ \\
\hline
\end{tabular}

Note:

- $\quad$ OF: organic fertilizer, BF: 2 L ha-1 biological fertilizer

- Values followed by different letters within a column are significantly different at $95 \%$ DMRT.

The yield components variables such as panicle length, number of grains per panicle, and 1000 grain weight on non fertilizer treatment was the lowest one, whereas in the second season two only the 1000 grain weight were influenced by fertilizers. The organic fertilizer was able to increase the weight of 1000 grains [13]. 
Table 4. Components of yield rice at different fertilizer treatments

\begin{tabular}{|c|c|c|c|c|c|}
\hline \multirow[t]{2}{*}{ Treatment } & $\begin{array}{l}\text { Number of } \\
\text { productive } \\
\text { tillers }\end{array}$ & $\begin{array}{l}\text { Panicle } \\
\text { length } \\
\text { (cm) }\end{array}$ & $\begin{array}{c}\text { Number of } \\
\text { grain per } \\
\text { panicle (grain) }\end{array}$ & $\begin{array}{c}1000 \\
\text { weight } \\
\text { grains }(\mathrm{g})\end{array}$ & $\begin{array}{c}\text { Empty } \\
\text { grain } \\
(\%)\end{array}$ \\
\hline & \multicolumn{5}{|c|}{ First season } \\
\hline 0 ton ha- ${ }^{1} \mathrm{OF}$ & $15.3 b$ & $24.1 \mathrm{cb}$ & $109.5 \mathrm{c}$ & $24.3 b$ & 5.6 \\
\hline 2 ton ha $^{-1} \mathrm{OF}$ & $16.3 \mathrm{ab}$ & $25.3 \mathrm{abc}$ & $132.8 \mathrm{abc}$ & $30.0 \mathrm{a}$ & 4.5 \\
\hline 4 ton ha- ${ }^{1} \mathrm{OF}$ & $17.2 \mathrm{ab}$ & $25.4 \mathrm{abc}$ & $133.1 \mathrm{abc}$ & $29.3 a$ & 4.9 \\
\hline 6 ton ha- ${ }^{-1} \mathrm{OF}$ & $16.8 \mathrm{ab}$ & $25.4 \mathrm{abc}$ & $131.5 \mathrm{abc}$ & $30.0 \mathrm{a}$ & 5.4 \\
\hline 8 ton ha $^{-1} \mathrm{OF}$ & $14.6 \mathrm{~b}$ & $24.9 \mathrm{abc}$ & $136.5 \mathrm{ab}$ & $30.0 \mathrm{a}$ & 6.7 \\
\hline 10 ton ha- ${ }^{1}$ OF & $15.5 b$ & $24.9 \mathrm{abc}$ & $132.5 \mathrm{abc}$ & $30.0 \mathrm{a}$ & 4.5 \\
\hline 0 ton ha- ${ }^{1} \mathrm{OF}+\mathrm{BF}$ & $17.8 \mathrm{ab}$ & $23.1 \mathrm{c}$ & $111.3 \mathrm{bc}$ & $31.0 \mathrm{a}$ & 4.9 \\
\hline 2 ton ha- ${ }^{1} \mathrm{OF}+\mathrm{BF}$ & $14.8 \mathrm{~b}$ & $23.8 \mathrm{bc}$ & $120.0 \mathrm{abc}$ & $30.0 \mathrm{a}$ & 5.5 \\
\hline 4 ton ha- $^{-1} \mathrm{OF}+\mathrm{BF}$ & $15.2 b$ & $24.3 \mathrm{bc}$ & $125.2 \mathrm{abc}$ & $29.7 \mathrm{a}$ & 4.3 \\
\hline 6 ton ha- ${ }^{-1} \mathrm{OF}+\mathrm{BF}$ & $14.9 \mathrm{~b}$ & $24.9 \mathrm{abc}$ & $134.0 \mathrm{abc}$ & $30.0 \mathrm{a}$ & 4.9 \\
\hline 8 ton ha- ${ }^{1} \mathrm{OF}+\mathrm{BF}$ & $15.9 \mathrm{ab}$ & $25.8 \mathrm{ab}$ & $137.7 \mathrm{a}$ & $30.0 \mathrm{a}$ & 5.1 \\
\hline 10 ton ha- ${ }^{1} \mathrm{OF}+\mathrm{BF}$ & $16.3 \mathrm{ab}$ & $26.7 \mathrm{a}$ & $142.2 \mathrm{a}$ & $29.7 a$ & 4.9 \\
\hline \multirow[t]{2}{*}{ Inorganic } & $19.6 \mathrm{a}$ & $24.8 \mathrm{abc}$ & $133.1 \mathrm{abc}$ & $29.3 \mathrm{a}$ & 4.2 \\
\hline & \multicolumn{5}{|c|}{ Second season } \\
\hline 0 ton ha- ${ }^{1} \mathrm{OF}$ & 17.9 & 28.8 & 89.2 & $28.79 \mathrm{ab}$ & 5.52 \\
\hline 2 ton ha- ${ }^{1} \mathrm{OF}$ & 16.3 & 21.3 & 92.1 & $31.66 \mathrm{ab}$ & 3.20 \\
\hline 4 ton ha- ${ }^{1} \mathrm{OF}$ & 14.7 & 23.2 & 105.0 & $31.19 \mathrm{ab}$ & 3.38 \\
\hline 6 ton ha- ${ }^{1} \mathrm{OF}$ & 16.8 & 23.1 & 101.9 & $33.41 \mathrm{ab}$ & 3.14 \\
\hline 8 ton ha- ${ }^{1} \mathrm{OF}$ & 15.5 & 23.0 & 104.2 & $31.58 \mathrm{ab}$ & 4.68 \\
\hline 10 ton ha- ${ }^{1} \mathrm{OF}$ & 13.7 & 23.3 & 109.3 & $30.79 \mathrm{ab}$ & 2.16 \\
\hline 0 ton ha $^{-1} \mathrm{OF}+\mathrm{BF}$ & 13.3 & 22.2 & 93.6 & $27.92 b$ & 2.85 \\
\hline 2 ton ha- ${ }^{1} \mathrm{OF}+\mathrm{BF}$ & 15.4 & 22.1 & 99.4 & $27.97 \mathrm{~b}$ & 3.65 \\
\hline 4 ton ha- ${ }^{1} \mathrm{OF}+\mathrm{BF}$ & 15.7 & 22.7 & 100.1 & $28.86 \mathrm{ab}$ & 2.26 \\
\hline 6 ton ha- ${ }^{1} \mathrm{OF}+\mathrm{BF}$ & 14.1 & 23.1 & 127.0 & $31.62 \mathrm{ab}$ & 2.54 \\
\hline 8 ton ha- ${ }^{1} \mathrm{OF}+\mathrm{BF}$ & 13.2 & 23.3 & 107.3 & $33.60 \mathrm{a}$ & 2.38 \\
\hline 10 ton ha- ${ }^{1} \mathrm{OF}+\mathrm{BF}$ & 15.2 & 22.5 & 104.2 & $31.95 \mathrm{ab}$ & 3.08 \\
\hline Inorganic & 15.4 & 29.7 & 105.5 & $31.29 \mathrm{ab}$ & 5.55 \\
\hline
\end{tabular}

Note:

- OF: organic fertilizer, BF: 2 L ha-1 biological fertilizer

- Values followed by different letters within a column are significantly different at $95 \%$ DMRT. 


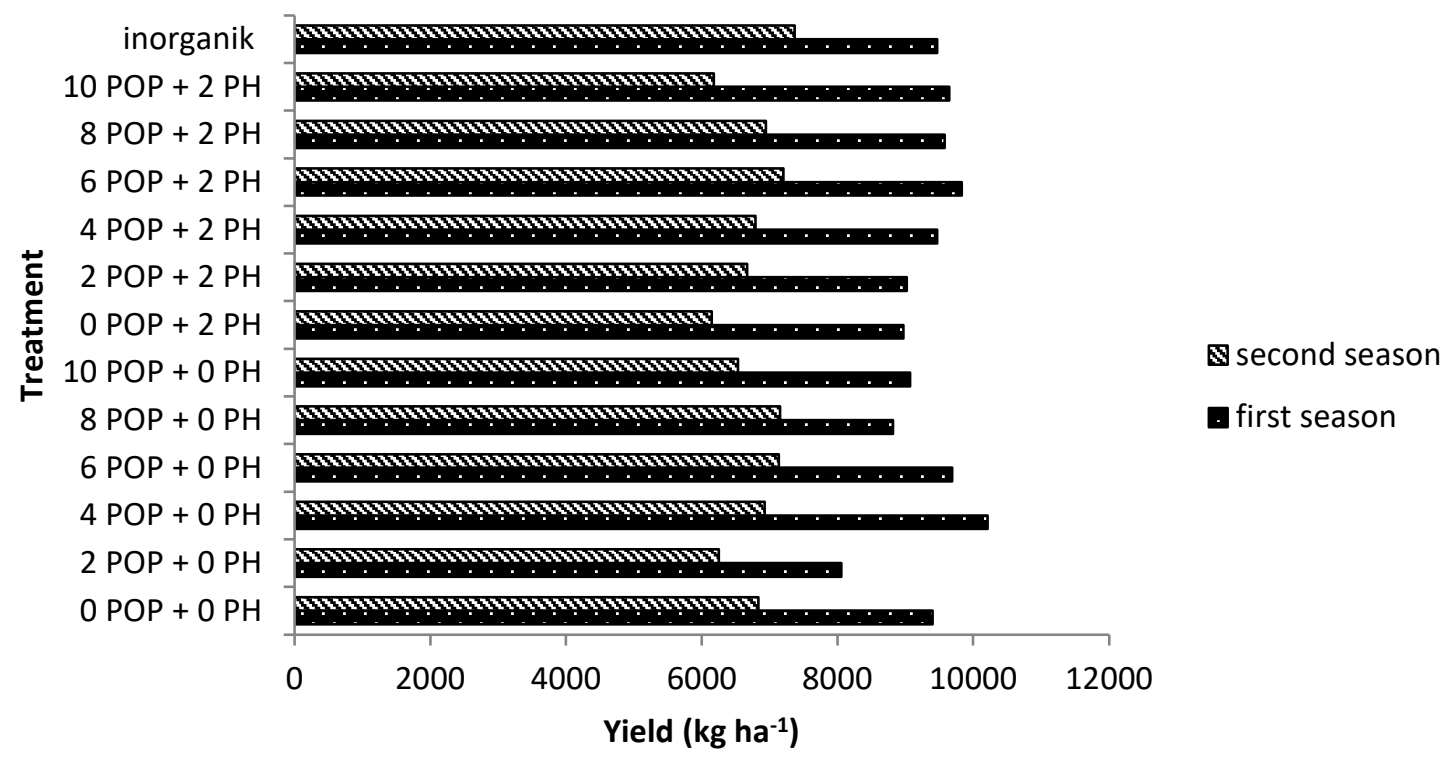

Figure 1. Yield in first and second season

Table 5. The NPK Levels of leaf and grain on different fertilizer treatment

\begin{tabular}{|c|c|c|c|c|c|c|}
\hline \multirow{2}{*}{ Treatment } & \multicolumn{3}{|c|}{ Leaf (\%) } & \multicolumn{3}{|c|}{ Grain (\%) } \\
\hline & $\mathbf{N}$ & $\mathbf{P}$ & $\mathbf{K}$ & $\mathbf{N}$ & $\mathbf{P}$ & $\mathbf{K}$ \\
\hline 0 ton ha- ${ }^{1} \mathrm{OF}$ & $1.32(\mathrm{D})$ & $0.27(\mathrm{O})$ & $1.69(\mathrm{O})$ & $1.19(\mathrm{~L})$ & $0.38(\mathrm{E})$ & $0.30(\mathrm{VL})$ \\
\hline 2 ton ha- ${ }^{1} \mathrm{OF}$ & $1.36(\mathrm{D})$ & $0.32(\mathrm{O})$ & $1.52(\mathrm{O})$ & $1.19(\mathrm{~L})$ & $0.39(\mathrm{E})$ & $0.28(\mathrm{VL})$ \\
\hline 4 ton ha- ${ }^{1} \mathrm{OF}$ & 1.41 (D) & $0.33(\mathrm{O})$ & $1.61(\mathrm{O})$ & $1.24(\mathrm{~L})$ & $0.40(\mathrm{E})$ & $0.30(\mathrm{VL})$ \\
\hline 6 ton ha- ${ }^{1} \mathrm{OF}$ & 1.18 (D) & $0.33(\mathrm{O})$ & $1.62(\mathrm{O})$ & $1.23(\mathrm{~L})$ & $0.40(\mathrm{E})$ & $0.32(\mathrm{VL})$ \\
\hline 8 ton ha- ${ }^{1} \mathrm{OF}$ & 1.50 (D) & $0.34(\mathrm{O})$ & $1.54(\mathrm{O})$ & $1.14(\mathrm{~L})$ & $0.38(\mathrm{E})$ & $0.31(\mathrm{VL})$ \\
\hline 10 ton ha- ${ }^{1} \mathrm{OF}$ & $1.26(\mathrm{D})$ & $0.34(\mathrm{O})$ & $1.60(\mathrm{O})$ & $1.18(\mathrm{~L})$ & $0.40(\mathrm{E})$ & $0.41(\mathrm{VL})$ \\
\hline 0 ton ha- ${ }^{1} \mathrm{OF}+\mathrm{BF}$ & $1.22(\mathrm{D})$ & $0.34(\mathrm{O})$ & $1.41(\mathrm{O})$ & $1.11(\mathrm{~L})$ & $0.40(\mathrm{E})$ & $0.38(\mathrm{VL})$ \\
\hline 2 ton ha- $^{-1} \mathrm{OF}+\mathrm{BF}$ & 1.37 (D) & $0.35(\mathrm{O})$ & $1.45(\mathrm{O})$ & $1.16(\mathrm{~L})$ & $0.40(\mathrm{E})$ & $0.37(\mathrm{VL})$ \\
\hline 4 ton ha- ${ }^{1} \mathrm{OF}+\mathrm{BF}$ & 1.20 (D) & $0.34(\mathrm{O})$ & $1.53(\mathrm{O})$ & $1.20(\mathrm{~L})$ & $0.39(\mathrm{E})$ & $0.36(\mathrm{VL})$ \\
\hline 6 ton ha- ${ }^{1} \mathrm{OF}+\mathrm{BF}$ & 1.15 (D) & $0.29(\mathrm{O})$ & $1.52(\mathrm{O})$ & $1.22(\mathrm{~L})$ & $0.40(\mathrm{E})$ & 0.50 (VL) \\
\hline 8 ton ha- ${ }^{1} \mathrm{OF}+\mathrm{BF}$ & 1.40 (D) & $0.30(\mathrm{O})$ & $1.58(\mathrm{O})$ & $1.18(\mathrm{~L})$ & $0.39(\mathrm{E})$ & $0.43(\mathrm{VL})$ \\
\hline 10 ton ha- ${ }^{1} \mathrm{OF}+\mathrm{BF}$ & 1.25 (D) & $0.30(\mathrm{O})$ & $1.50(\mathrm{O})$ & $1.15(\mathrm{~L})$ & $0.39(\mathrm{E})$ & $0.40(\mathrm{VL})$ \\
\hline Inorganic & $1.32(\mathrm{D})$ & $0.28(\mathrm{O})$ & $1.74(\mathrm{O})$ & $1.31(\mathrm{~L})$ & $0.40(\mathrm{E})$ & 0.34 (VL) \\
\hline
\end{tabular}
Note:

OF: organic fertilizer, BF: 2 L ha-1 biological fertilize

(D): nutrient deficiency, $(\mathrm{O})$ : optimum, $(\mathrm{L})$ : limited, $(\mathrm{SL})$ : very limited, $(\mathrm{E})$ : excess 
Figure 1 showed that the yield per hectare was not affected by fertilizer treatment either in the first season and second season. It showed that the wet yield per hectare in the second season was lower than the first season. It was suspected that the first season was existence of nutrients stored in the soil. This is indicated by the $\mathrm{P}$ is very high before first season and decreased in the first and second season (Table 1).

\section{Discussion}

Decrease in soil $\mathrm{pH}$ can occur due to decomposition of organic materials provided on the ground can produce organic acids [14]. A decreasing in soil $\mathrm{pH}$ can occur as a result of organic matter in the soil weathering given to the role of microorganisms that produce plant nutrients, organic acids, CO2 and energy [15]. In general, the levels of N-total at the higher end of the study compared to prior studies, except in the treatment of 8 tons of organic fertilizer ha- $1+2$ $\mathrm{L}$ ha-1 biological fertilizer. The increased levels of $\mathrm{N}$ and $\mathrm{K}$ in the soil experiment is mainly due to the possibility of the addition of $\mathrm{N}$ and $\mathrm{K}$ from organic fertilizers are slow available. One of the properties of the organic material is slow to provide nutrients [12].

Based on the analysis of $\mathrm{pH}$, the soil in the sour category, so there was the possibility of soil acidity resulting in a low $\mathrm{P}$ availability. In acid soils (low $\mathrm{pH}$ ) elements $\mathrm{Fe}$ and $\mathrm{Al}$ are dissolved in the soil will bind to $\mathrm{P}$ so that phosphorus becomes unavailable or availability of $\mathrm{P}$ will be reduced [16].

The leaf color scores in the treatment of inorganic fertilizers is higher than the organic fertilizer treatments. This is due to the slow nature of the organic matter provides nutrients [7]. Leaf color scores on all treatments showed less than 4 and $\mathrm{N}$ content of leaves showed criterion deficiency $(<2 \%)$ (Table 5). Leaf color value in all treatments remained below the critical point was $<4$ shows the inadequacy of nutrient $\mathrm{N}$ in plants [17]. In plants that fulfilled the needs of nitrogenous nutrients will have dark green leaves. Treatment without or with organic fertilizer biological fertilizer affects variables 1000 grain weight. Table 4 shows the weight of 1000 grains grow in line with increasing doses of organic fertilizer $(\mathrm{P}<0.05)$, with the highest 1000 grain weight in the treatment of 8 tons ha- 1 organic fertilizer + biofertilizer. The organic fertilizer can increase the weight of 1000 grains [13,18, 19,20]

Yield was not affected by the treatments because of the low availability of $\mathrm{N}$, which can be seen from the color of the leaves are small [21] as well as the nutrient content of $\mathrm{N}$ in the leaves are small. Based on the criteria of nutrient adequacy rice plants, organic rice crop deficiency of $\mathrm{N}$, but contains enough $\mathrm{P}$ and $\mathrm{K}$ (Figure 16) [22]. P and K content of leaves including sufficient criteria, but less leaf $\mathrm{N}$ content, thus making nitrogen becomes the limiting factor. The nitrogen effect on plant height, number of tillers and yield. The growth of the rice plant is determined by the adequacy of $\mathrm{N}$ and $\mathrm{P}$, whereas for root growth is determined by the adequacy of $\mathrm{P}[22]$.

\section{Conclussions}

The variables first season such as number of tillers and leaf color in inorganic fertilizer treatment was higher when compared with the organic fertilizers. Soil fertility increased in the second season in organic treatment and yield of rice decreased in the second season. 


\section{References}

[1] Lee, H.J., J. S. Lee, J. H. Seo. Decomposition and N realease of Hairy Vetch Applied as a Green manure and Its Effect on rice yield in paddy fields. Korean Journal of Crop Science 47 (2): 137-141. (2002)

[2] Sisworo, WR food self-sufficiency and sustainable agriculture, the challenges of the XXI century. Approach to soil science, plant, and utilization of nuclear science and technology. BATAN, Jakarta. (2006)

[3] Faizal, M. Analysis of organic rice marketing; cases superior marketing of organic rice boom in the West Java city of Bogor. Nusa Farmers Journal 9 (2): 45-55. (2009)

[4] Hota R., Jena A.K., Narayana K. L. Effect of Inorganic and Organic Amendments on Yield of Cocoyam (Colocasia esculenta), and on Soil Properties. World Journal of Agricultural Research 2 (2): 70-81. (2014).

[5] Cyio, M.B. Effectiveness of organic material and water level to changes in Eh, $\mathrm{pH}$, and the status of Fe, P, Al, dissolved in soil ultisol. Agroland Journal 15 (4): 257-263. (2008).

[6] Hadi, P., Sarwono. Effect of organic fertilizers and pesticides on the growth and yield of black rice. Journal of Agricultural Innovation 11 (1): 60-69. (2013)

[7] Arafat, M. P. Sirappa. Study of Use of straw and fertilizer N, P, and K on irrigated land. Journal of Soil and Environmental Sciences 4 (1): 15-24. (2003).

[8] Zahrah, S. bokhasi and NPK fertilizer application on soil organic ultisol for rice crops with a system of SRI (System of Rice Intensification). Journal of Environmental Science 5 (2): 114-129. (2011).

[9] Agung T., A. Y. Rahayu. Efficiency Analysis of N uptake, Growth, and Yields of Several New Superior Soybean Cultivars by Drought Stress and Biological Fertilizers. Agrosains 6 (2): 70-74. (2004).

[10] Las, I. Tim. Land Resource and climate supports sustainable self-sufficiency in rice. Memiograf. Center for Research and Development of Agricultural Land Resources, Bogor. (2008).

[11] Syamsudin, T.S., S. Aktaviyani. Penerapan pemupukan pada pertanian padi organic dengan metode system of rice intensification (SRI) di desa Sukakarsa kabupaten Tasikmalaya. J. Agroland 16 [1] : $1-8 .(2009)$

[12] Suharno, A.K. Rachman, S.R. Apsari. Effect of organic fertilizer types on the growth and production of sweet potatoes (Ipomea batatas L.). Agriekstensia 9 (2): 200-210. (2010).

[13] Siregar, D., Marbun, P., Marpaung, P. Effect of varieties and different organic materials to 1000 grains and paddy rice biomass IP 400 on the season Agroecotechnology I. Online Journal 1 (4): 1413 1421. (2013)

[14] Sugito Y, Yulia N, Ellis N. Organic Agriculture Systems. Malang (ID): Faculty of Agriculture, Brawijaya University. (1995).

[15Chairani. Effect of phosphorus and cow manure on the soil chemical properties and growth of rice plants (Oryza sativa) on rainfed lowland in Langkat District, North Sumatra. Journal of Agricultural Research 25 (1): pp. 8-17. (2006).

[16] Herviyanti, Ahmad F., Sofyani R, Darmawan, Gusnidar, Saidi A., Effect of humic material from young coal extract (Subbituminus) and $\mathrm{P}$ fertilizer on the chemical properties of ultisol and the production of corn (Zea mays L.) J. Solum 9 (1): 15-24. (2012).

[17] Wahid, A. S. Increased efficiency of nitrogen fertilizer in lowland rice using the leaf color chart method. Agricultural Research and Development Journal. 22 (4): 156-161. (2003).

[18] Pramono E. Effect of organic fertilizer and micro fertilizer on the production of quality rice seeds (Oryza sativa L.). Agronomics 10 (1): 11-22. (2010)

[19]Wangiyana W, Budiyanto VFA, Farida N, Dulur NWD. Growth and yield of rice (Oryza sativa L.) v Silugonggo in various cultivation techniques and application of compost bokhasi cow manure. Agroteksos 20 (3): 103-111. (2010).

[20] Soplanit R, Nukuhaly SH. Effect of NPK nutrient management on the availability of N and yields of lowland rice in Waelo village, Waeapo sub-district, Buru district. Agrologia 1 (1): 81-90. (2012)

[21] Nurmayulis, P. Utama, D. Firnia, H. Yani, A. Citraresmini. The response of nitrogen and azolla to the growth of Mira I variety rice plants with the SRI method. Scientific Journal of Isotope and Radiation Applications 7 (2): 115-130. (2011) 
[22] Dobermann,A. and T.H. Fairhurst. In Rice Nutrient Disorders and Nutrient Management.IRRI/PPIC pp.3. (2000) 